\title{
On the detection of ultra high energy neutrinos with the Auger observatory
}

\author{
K.S. Capelle ${ }^{\mathrm{a}}$, J.W. Cronin ${ }^{\mathrm{a}}$, G. Parente ${ }^{\mathrm{b}}$, E. Zas ${ }^{\mathrm{b}}$ \\ ${ }^{a}$ Department of Physics and Enrico Fermi Institute, University of Chicago, Chicago, IL 60637, USA \\ ${ }^{\mathrm{b}}$ Departamento de Física de Partículas, Universidade de Santiago de Compostela, E-15706 Santiago, Spain
}

Received 16 May 1997; revised 3 October 1997

\begin{abstract}
We show that the Auger Air Shower Array has the potential to detect neutrinos of energies in the $10^{19} \mathrm{eV}$ range through horizontal air showers. Assuming some simple conservative trigger requirements, we obtain the acceptance for horizontal air showers as induced by high energy neutrinos by two alternative methods and we then give the expected event rates for a variety of neutrino fluxes as predicted in different models which are used for reference. (c) 1998 Elsevier Science B.V.
\end{abstract}

PACS: 95.85.Ry; 96.40.Tv; 96.40.Pq; 98.70.Sa

\section{Introduction}

High energy neutrino detection is one of the experimental challenges in particle astrophysics for the forthcoming years because it opens a new window to the regions of the Universe that are otherwise shielded from us by large amounts of matter. It is widely believed that one of the most appropriate techniques for neutrino detection consists of detecting the Čerenkov light from muons or showers produced by the neutrino interactions in underground water or ice. This allows the instrumentation of large enough volumes to compensate for both the low neutrino cross section and the low fluxes expected. There are several projects under way to build sufficiently large detectors to measure the expected signals from a variety of sources [1].

On the other hand, many years ago it was suggested that deeply penetrating high energy particles, such as muons and neutrinos, initiate large horizontal air showers that can be detected at ground level [2].
At large zenith angles the electromagnetic part of ordinary air showers, initiated by cosmic rays of hadronic (or electromagnetic) nature, is attenuated by the atmosphere well before reaching ground level. This should allow the identification of the showers initiated by these deeply penetrating particles.

Recently, there has been a proposal to build two $3000 \mathrm{~km}^{2}$ extensive air shower arrays, one in each hemisphere, to detect cosmic rays with energy above $10^{19} \mathrm{eV}$ (the Pierre Auger project) [3]. Each array consists of a hexagonal grid of water tanks, $3.5 \mathrm{~m}$ diameter and $1.2 \mathrm{~m}$ height, combined with an optical fluorescence detector. The tanks are separated $1.5 \mathrm{~km}$ from each other and instrumented with photodetectors to detect the Čerenkov light emitted as photons and charged particles from the shower front cross it. We will show that these detectors can also play an important and complementary role for detecting neutrinos of energy around $10^{19} \mathrm{eV}$ and above.

Neutrinos of these energies cannot penetrate the 
earth so any detector searching them must look for events with zenith angles between near horizontal and vertical downgoing. For showers of sufficient energy the array efficiency is high and the low target density for neutrinos provided by the atmosphere is compensated by the huge surface area of the planned array. The observatory will complement other neutrino detectors in construction or planning.

In this article we show that the Pierre Auger Observatories have an enormous potential to detect neutrinos in the EeV range. In Section 2 we firstly present some of the possible $\mathrm{EeV}$ neutrino fluxes that have been discussed in the literature which will be used later in the evaluation of the event rates expected by the Auger particle arrays. In Section 3 we discuss the acceptance for the horizontal air showers. Firstly we obtain a rough but intuitive analytical expression for the acceptance of a large array, which we discuss in the context of the Pierre Auger project. In Section 3.1 we present an acceptance calculation for the Auger Project based on a geometrical approach. In Section 3.2 an alternative and conservative calculation based on Monte Carlo simulation is presented. In Section 4 we estimate the horizontal shower rates expected for the neutrino fluxes addressed in Section 2, and Section 5 is reserved for the conclusions.

We do not attempt to discuss event reconstruction or backgrounds which will be addressed elsewhere.

\section{EeV neutrino fluxes}

Neutrinos of EeV energies are likely to be directly produced together with cosmic rays of the highest energies whose origin is still a matter of speculation. Several possible sources of neutrinos in the EeV region have been discussed in this context. Moreover, provided that the highest energy cosmic rays are extragalactic in origin, as it is currently believed, EeV neutrinos have to be produced in their interactions with cosmic microwave photons. We will use some of the neutrino flux predictions reported in the literature as reference calculations to evaluate the event rates that could be expected in each Auger Observatory. In this section, we briefly motivate these fluxes which are shown in Fig. 1 compared with the expected atmospheric neutrino flux.

Active Galactic Nuclei (AGN), the most ener-

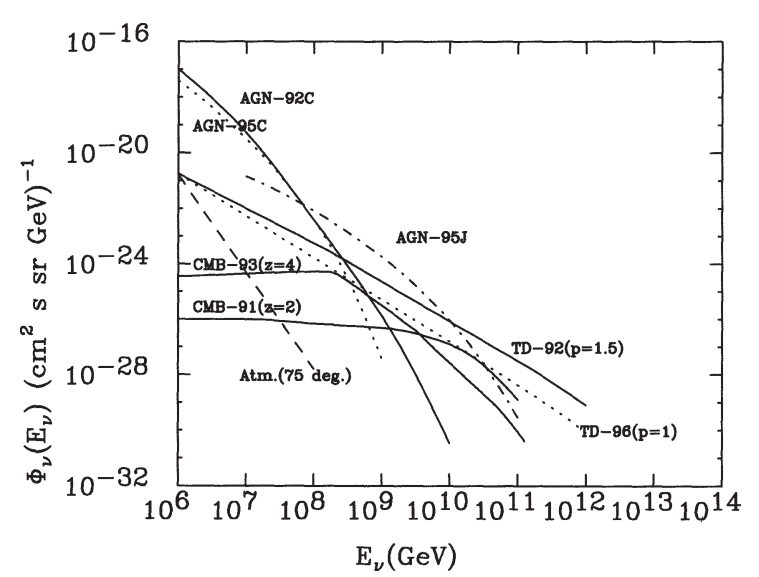

Fig. 1. Neutrino flux predictions in the $\mathrm{EeV}$ range as labeled in the text.

getic objects known, emit most of their luminosity in gamma rays [4] and may be the source of the highest energy cosmic rays [5]. Multiwavelength observation of these sources leads us to believe they are powered by large accreting black holes, where shock fronts provide particle acceleration. If, besides electrons, protons are also accelerated, as some models suggest, photoproduction of pions with the ambient light plays a crucial role (see for example Ref. [6] and references therein). The energetic gamma rays observed come from the decays of $\pi^{0}$ 's and high energy neutrinos from the decays of charged pions become a signature of these models. The first models for neutrino production in AGN assumed shock acceleration in the AGN cores and predicted relatively flat fluxes up to energies of about $10^{15} \mathrm{eV}$. For our event rate calculation we select the prediction of Ref. [7], labelled AGN-92C, which is quite similar to that in Ref. [6] (AGN-95C).

There is however recent evidence that the $\mathrm{GeV}$ to $\mathrm{TeV}$ gamma ray emission observed from AGN corresponds to the blazar class [8]. In a unified AGN description, the blazar class is identified as AGN with jets of ultrarelativistic particles streaming out of their cores and pointing towards us. Most recent models for the proton blazars site the acceleration in the jets themselves. Photoproduced neutrinos are Lorentz boosted to energies well above those predicted in the models of acceleration in the AGN cores. We use the prediction of Ref. [9] (labelled AGN-95J) which illustrates that the emitted neutrinos may extend well into the 
EeV region in agreement with Ref. [10].

We also consider the more speculative and uncertain models where the highest energy cosmic rays are produced by the decays of topological defects. These objects emit massive X particles, predicted in Grand Unified theories, which decay and fragment into Standard Model particles. There are a fair number of neutrino flux predictions from these models depending on the different time evolution of the effective injection rate of $\mathrm{X}$ particles per unit volume (usually denoted with a parameter $p$ ), the masses of the X particles themselves and other unknown parameters. Some models have already been constrained by bounds on horizontal showers induced by neutrinos [11] and also by measurements of the $100 \mathrm{MeV}$ diffuse gamma ray background [12]. We select two fluxes illustrating the range of predictions in such models. We use the prediction with $p=1.5$ in Ref. [13] (labelled TD-92) and that given in Ref. [14] which is labelled as TD96. TD-92 is the lowest neutrino prediction given in Ref. [13] and is not severely affected by experimental constraints.

Lastly we consider two predictions for the high energy neutrinos produced in the interactions of cosmic rays with the cosmic microwave background: CMB91 [15] and CMB-93 corresponding to model 4 in Ref. [16]. They illustrate the variations that can be associated to various uncertainties intrinsic to these calculations. These neutrinos are a direct result of the Greisen-Zatsepin-Kuz'min cutoff and must be produced if the highest energy cosmic rays are of extragalactic origin. Unfortunately it is possible that the flux levels are too low to be observed either with the Pierre Auger Observatory or with other neutrino detectors in planning or construction.

When the electron-neutrino flux prediction is not explicitly available in any of the models used, we approximate it to be a factor of two below the muon neutrino flux as expected by naive channel counting in pion production and decay.

\section{Acceptance for neutrino showers}

Neutrinos produce showers in most interactions with the atmosphere which are of different nature depending on the process in consideration. We consider both deep inelastic charged and neutral current inter- actions which always produce hadronic type showers. In the case of charged current electron-neutrino interactions the emerging electron contributes in addition a pure electromagnetic shower carrying a large fraction of the incoming particle energy. We will ignore the resonant cross section because it is only significant near the peak of the cross section which occurs at an incoming neutrino energy of $6.4 \mathrm{PeV}$, well below the region of high efficiency for the Pierre Auger project.

For a neutrino flux $d \Phi_{\nu} / d E_{\nu}$ interacting through a process with differential cross section $d \sigma / d y$, where $y$ is the fraction of the incident particle energy transferred to the target, the event rate for horizontal showers can be obtained by a simple convolution,

$$
\begin{aligned}
& \Phi_{\mathrm{sh}} {\left[E_{\mathrm{sh}}>E_{\mathrm{th}}\right]=N_{a} \rho_{\mathrm{air}} \int_{E_{\mathrm{th}}}^{\infty} d E_{\mathrm{sh}} } \\
& \times \int_{0}^{1} d y \frac{d \Phi_{\nu}}{d E_{\nu}}\left(E_{\nu}\right) \frac{d \sigma}{d y}\left(E_{\nu}, y\right) \mathcal{A}\left(y, E_{\nu}\right),
\end{aligned}
$$

where $N_{a}$ is Avogadro's number and $\rho_{\text {air }}$ is the air density. The energy integral corresponds to the shower energy $E_{\mathrm{sh}}$ which is related to the primary neutrino energy $E_{\nu}$ in a different way depending on the interaction being considered. $\mathcal{A}$ is a geometric acceptance, a function of shower energy, which corresponds to the volume and solid angle integrals for different shower positions and orientations with respect to the array. The function is different for showers induced by charged current electron-neutrino interactions from those arising in neutral current or muon-neutrino interactions. This is because hadronic and electromagnetic showers have differences in the particle distributions functions, particularly for muons.

For showers of sufficient energy, incident with zenith angle $\theta_{\text {zenith }}$, the effective volume for horizontal shower detection is given by $S \cos \theta_{\text {zenith }}$, where $S$ is the surface area covered by the array, multiplied by the range of allowed positions for the first interaction point along the incident direction. We can estimate this range approximating shower maximum as a disk of some effective radius $r$. This radius should approximately be the maximum distance to the shower axis at which the particle density is high enough to trigger the detector tanks (see below). As the first interaction point is moved along the incident direction, 


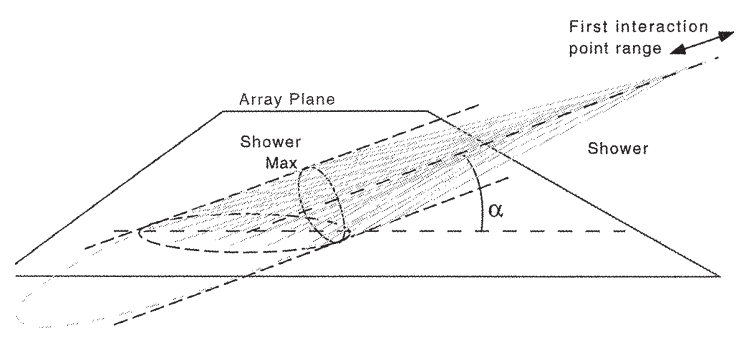

Fig. 2. Schematic picture of a horizontal shower, illustrating the disk of radius $r$ at shower maximum and the cylinder it spans as the first interaction point is shifted along the incident direction. The intersection of the cylinder is an ellipse of major axis $q$ (see text).

the intersection of this disk with the detector plane is an ellipse with major axis $q=2 r / \sin \alpha$ with $\alpha=$ $90^{\circ}-\theta_{\text {zenith }}$. The projection of this axis onto shower direction $(q \cos \alpha)$ is the wanted range (see Fig. 2).

We estimate $\mathcal{A}$ integrating this volume over the possible solid angle orientations of the shower, $d \Omega=$ $2 \pi d(\sin \alpha)$, and restrict the integration to horizontal showers, i.e. $0^{\circ}<\alpha<\alpha_{\max } \simeq 15^{\circ}$. Approximating $\cos \alpha \simeq 1$, we obtain a simple analytic expression in terms of $r$,

$$
\begin{aligned}
\mathcal{A}= & S \times 2 \pi\left(\int_{\sin \alpha_{1}}^{\sin \alpha_{\max }} d(\sin \alpha) \sin \alpha \frac{2 r}{\sin \alpha}\right. \\
& \left.+\int_{0}^{\sin \alpha_{1}} d(\sin \alpha) \sin \alpha \widehat{W}\right) \\
= & S \times 2 \pi r\left(2 \sin \alpha_{\max }-\sin \alpha_{1}\right) .
\end{aligned}
$$

For $\alpha<\alpha_{1}=\sin ^{-1}(2 r / \widehat{W})$, the ellipse axis $q$ exceeds $\widehat{W}$, the average size of the array ${ }^{1}$. The last term in the brackets of Eq. (2) represents a small correction obtained when restricting the range of the interaction point to be below the longitudinal size of the array. Most importantly, the acceptance is seen to depend on the array surface area and the shower radius. The estimate makes the assumption that showers with shower maximum intercepting the array are detected with close to $100 \%$ efficiency. The effective radius has to be chosen to match this requirement which will only hold for energies above a given threshold. It is conservative in the sense that it ignores the fact that show-

\footnotetext{
${ }^{1}$ For $S=3000 \mathrm{~km}^{2}$, the "diameter" of the Pierre Auger array is approximately $D=65 \mathrm{~km}$ and $\widehat{W} \simeq 0.70 D \sim 45 \mathrm{~km}$.
}

ers can trigger the detector without having shower maximum intercepting the array, or even without the shower axis going through the array (i.e., completely horizontal showers).

It is easy to see that the acceptance for the Auger particle arrays is comparable to other neutrino detectors in planning [17]. Since design requirements have led to a tank size that allows near $100 \%$ efficiency for vertical showers of energy above $10^{19} \mathrm{eV}$ [3], the expected signal in the tanks should be large enough to trigger up to distances from shower axis of the order of the separation between the tanks, $1.5 \mathrm{~km}$. It is thus conservative to expect similar efficiencies for horizontal showers above $10^{19} \mathrm{eV}$ as they should not differ that much from vertical ones at detector level and to approximate $r \sim 1.5 \mathrm{~km}$. The water Čerenkov technique is particularly well suited for horizontal showers. The transverse separation between detectors will be substantially reduced for near horizontal showers and the extra depth of the tanks in the horizontal mode should enhance the signal from the muons in the shower, compensating the reduction in transverse area of the tank to the incident direction.

Taking $r=1.5 \mathrm{~km}\left(\alpha_{1}=4^{0}\right)$, we obtain an estimate for the Auger array acceptance of showers of energy above $\sim 10^{19} \mathrm{eV}, \mathcal{A}=13000 \mathrm{~km}^{3}$ sr which when multiplied by an air density $\rho_{\text {air }} \simeq 10^{-3} \mathrm{~g} \mathrm{~cm}^{-3}$ gives $1.3 \times 10^{7} k T$ sr. Underground high energy neutrino detectors in planning aim towards an active volume in the range of $1 \mathrm{~km}^{3}$ of water [18]. In models where most of the events are due to neutrinos well above the $\mathrm{PeV}$ region, the Earth will be opaque to them. The corresponding acceptance for contained events is of order $6 \times 10^{6} k T \mathrm{sr}^{2}$, illustrating how the Pierre Auger project may come into play.

The above estimate of the acceptance does not display the energy dependence but it illustrates how it depends on the effective shower radius, which in turn must depend on energy. As it happens for vertical showers, the acceptance must show a kind of threshold behavior, since sufficiently low energy showers cannot be detected with such a sparse array. The dependence of the acceptance on shower energy has been introduced in two alternative ways. In a more geomet-

\footnotetext{
${ }^{2}$ However, for muon neutrinos the long range of the muon increases the acceptance for non-contained charged current interactions.
} 
rical approach we have followed on the above line of thinking considering in more detail the shower depth development and the lateral distribution functions for electrons and positrons. As an alternative we have simulated showers at different positions with respect to the detector in a Monte Carlo approach.

\subsection{Geometrical approach}

The calculation treats electromagnetic and hadronic cascades independently using the conventional NKG charged particle lateral distribution functions [19] normalized with the Gaisser [20] (Greisen [19]) parametrization for the total number of electrons and positrons in hadronic (electromagnetic) cascades.

We assume that triggering requirements for each tank can be specified as fixed numbers for the electron density in the electromagnetic and hadronic showers: $\rho_{e}^{\text {th }}$. The relevant quantity for the tank signal is the Cerenkov light, proportional to the charged particle tracklength, which is in turn approximately proportional to energy deposition. Muons travel through the tank depositing about $2 \mathrm{MeV} /\left(\mathrm{g} \mathrm{cm}^{-2}\right)$ while most electrons and photons are stopped in the tanks, depositing all their energy. We use $\rho_{e}^{\text {th }}$ because there are convenient parameterizations in the literature for this quantity. The relation between $\rho_{e}^{\text {th }}$ and energy deposition is however dependent on the shower position and can only be done in an approximate manner.

The above assumption naturally defines the active region of a shower, the volume within which the electron density exceeds the given threshold $\rho_{e}^{\text {th }}$. The region is bounded by a cigar shaped contour plot of the three-dimensional electron distribution function at $\rho_{e}^{\text {th }}$. For the solid angle-volume acceptance integration, we consider the number of tanks contained in the intersection of these active volumes with the detector plane as the shower directions and first interaction point are varied. The electron-positron density at these tanks is by definition above threshold. At typical large distances (of order a kilometer) to the shower axis, we estimate that an electron density in the range 0.6$1.2 \mathrm{~m}^{-2}\left(0.3-0.5 \mathrm{~m}^{-2}\right)$ for horizontal electromagnetic (hadronic) showers is equivalent to an energy deposition of $500 \mathrm{MeV}$ in a tank (corresponding to about two vertical muons). This is considered sufficient for shower detection [3].

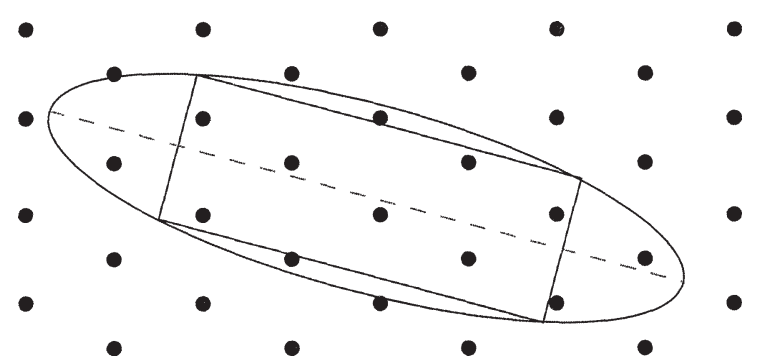

Fig. 3. Illustration of the intersection of the "active part" of the shower (as described in the text) and the detector plane used in the geometrical approach. The intersection, which is close to an ellipse, is approximated by a rectangle as illustrated. The dots correspond to the detector tanks.

Approximating the intersections of these active regions as rectangles allows a complex but analytical solution to the problem if the only orientations and positions of the rectangles considered in the integration are those which contain at least $n$ tanks in the same row (see Fig. 3). As a result we obtain the acceptance curves as a function of shower energy for each shower distribution function which only depend on the parameter $\rho_{e}^{\text {th }}$. The important advantage of this calculational method against the obvious alternative which involves Monte Carlo simulation (addressed below) is computing speed. The approach converts in straightforward the otherwise lengthy evaluation of the effect of changing the input parameters in the calculation such as trigger conditions, tank thresholds, parameterizations of shower distribution functions and even array spacing.

In Fig. 4 we show the acceptance results for a trigger of at least $n=3$ aligned tanks, having the conservative electron density of $1.2 \mathrm{~m}^{-2}\left(0.5 \mathrm{~m}^{-2}\right)$ for electromagnetic (hadronic) showers and considering only showers with zenith angle higher than 75 degrees. For each shower type there are two curves, one considers showers with axis falling within the array and the second also includes an approximate calculation of the triggering showers with axis not going through the array. It is important to note that these showers make most of the contribution to the acceptance in the high energy limit (corresponding to huge showers flying parallel and on top of the array).

Besides using $\rho_{e}^{\text {th }}$ as the parameter for tank threshold, the geometrical algorithm described above has a potentially more important drawback. By using parameterizations for air showers, it ignores the effect of 


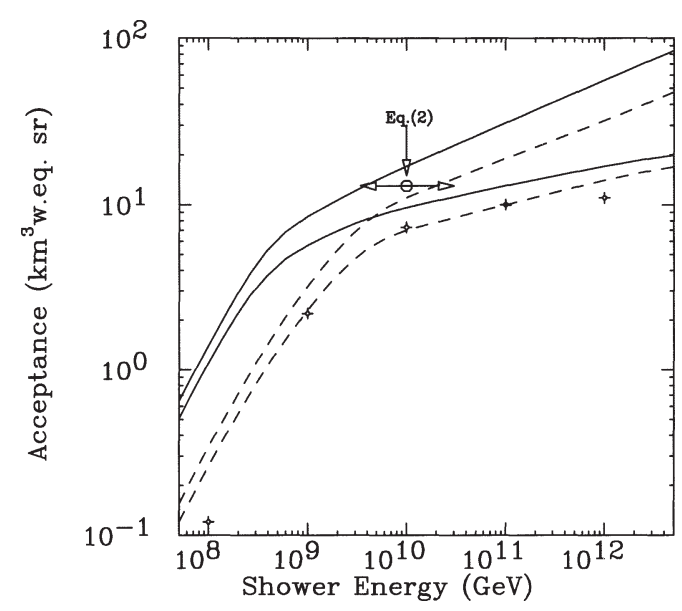

Fig. 4. Acceptance of the Pierre Auger detector to near horizontal showers $\left(\theta_{\text {zenith }} \geq 75\right.$ degrees $)$. Volume units are $\mathrm{km}^{3}$ of water equivalent. Crosses are for the results in the Monte Carlo approach, lines are correspond to the geometrical integration for electromagnetic showers (dashed) and hadronic (solid). The lower set of curves corresponds to only showers with axis falling in the array and the upper set takes all showers into consideration.

the ground on shower development. The simplification can be thought to grossly overestimate the acceptance. Using EGS4 with constant density air, we have simulated the effect of the ground for very inclined electromagnetic showers of energies up to $10^{15} \mathrm{eV}$. In spite of the reduction in the lateral distribution observed after the core hits the ground, there is only a very small reduction in the acceptance assuming the results can be extrapolated to higher energies. In any case we have decided to contrast the acceptance results against a totally different calculational approach, which we chose to remedy these drawbacks in a conservative manner. That way we hope to bracket a prediction for the horizontal shower acceptance of the Auger project.

\subsection{Monte Carlo approach}

We have performed a totally independent and conservative calculation with a hybrid Monte Carlo and parametrization method. The approach uses the simulated curves of energy deposition versus distance from shower axis in the Pierre Auger water tanks for a $10^{19} \mathrm{eV}$ shower at different depths. These results are then simply scaled with shower energy. We have restricted the range of depths for the shower to the interval $[500,1600] \mathrm{g} \mathrm{cm}^{-2}$ and we only consider showers with the shower axis falling within the array. In our effort to be conservative, we completely neglect the contributions from particles after the shower core hits the ground. We have then generated horizontal events by allowing the starting point and direction $\left(75^{\circ}<\right.$ $\theta_{\text {zenith }}<90^{\circ}$ ) of the shower to vary in a random way, only restricted by the array size and a maximum height of $3 \mathrm{~km}$.

In the simulation of the acceptance, events are detected when the shower deposits more than $500 \mathrm{MeV}$ of energy in each of four or more tanks. We have used an average parameterization assuming half of the energy induces a hadronic shower and the other half an electromagnetic shower. Besides correcting drawbacks in the geometrical approach, the method has the advantage of allowing the study of the different patterns of hit tanks on an event by event basis [21]. The results are shown as points in Fig. 4 for some discrete energies. Given the fundamental differences between the two approaches, it is not possible to test one against the other. Nevertheless, it is fair to remark that the agreement is rather good and that the differences of results can be interpreted on the basis of the different inputs intrinsic to each method. We thus take the Monte Carlo approach as a lower bound of the acceptance and the difference between the two approaches can be considered as an indication of the degree of uncertainty.

For the detection of the horizontal showers included in the acceptance calculations described here, only directional reconstruction and an ability to separate the electromagnetic part of the shower from the muonic component should be sufficient in principle. Nevertheless, if the long range of the muons produced in the horizontal showers of hadronic nature is also taken into account, it can be argued that the acceptance may exceed that actually calculated in this work. The muon component effectively enhances the length of the active region of the shower, what must increase the acceptance for deeply penetrating particles. The identification of such showers would become however more complicated, because they look more like those induced by cosmic rays at very large zenith angles. 
Table 1

Yearly neutrino event rates for different diffuse fluxes and cross sections (see text). The first entry corresponds to the upper set of curves in Fig. 2 (all showers) and the more conservative second entry is the result of the simulation. The last column gives the energy range of the bulk of the events. Events correspond to a single array of $3000 \mathrm{~km}^{2}$.

\begin{tabular}{llll}
\hline$\nu$ source & MRS(R1) & GRV 95 & Energy $(\mathrm{GeV})$ \\
\hline AGN-92C [7] & $2.5 / 0.4$ & $2 / 0.3$ & $10^{6}<E<10^{8}$ \\
AGN-95C [6] & $1.5 / 0.2$ & $1.2 / 0.2$ & $10^{6}<E<10^{8}$ \\
AGN-95J [9] & $7 / 2.1$ & $5.5 / 1.6$ & $10^{8}<E<10^{10}$ \\
CMB-91 [15] & $0.5 / 0.2$ & $0.4 / 0.1$ & $10^{10}<E<10^{11}$ \\
CMB-93 [16] & $0.2 / 0.06$ & $0.13 / 0.04$ & $10^{8}<E<10^{12}$ \\
TD-92 [13] & $12 / 4$ & $7 / 2.4$ & $10^{9}<E<10^{13}$ \\
TD-96 [14] & $1.4 / 0.5$ & $0.9 / 0.3$ & $10^{10}<E<10^{12}$ \\
\hline
\end{tabular}

\section{Event rates}

To calculate event rates, we use the deep inelastic charged and neutral current cross section from two sets of structure functions MRS(R1) and GRV ( $\overline{\mathrm{MS}}$ renormalization scheme) parton distributions [22]. With increasing energy, neutrinos interact with partons carrying a lower fraction $x$ of nucleon momentum which extends beyond the kinematical limits of the parton density parameterizations. For the first set we extrapolate to low $x$ beyond validity of the parameterization, using the slope of $x q(x)$, at the lowest $x$ permitted (in this case $x=10^{-5}$ ), where $q(x)$ is the standard parton distribution. This is consistent with extrapolations based on the leading log approximation [23]. The second set, GRV, can be cautiously used on its own for low $x$.

Table 1 displays the event rates for different neutrinos fluxes, calculated with Eq. (1) for each of the two cross sections. The first entry corresponds to the result of our conservative approach based on the simulation and the higher one to the acceptance results calculated in the geometrical approach including showers with axis not intercepting the array. Recent models of proton acceleration in AGN jets (AGN-95J) and some models of decays of topological defects ( such as TD92) predict neutrino fluxes giving measurable rates in the most conservative assumptions. For neutrinos from AGN cores the majority of the detected showers lie in the $10^{15}-10^{17} \mathrm{eV}$ region. This corresponds to the threshold region for the acceptance curves where the efficiency is low and are very sensitive to trigger conditions. For these neutrino fluxes, a $1 \mathrm{~km}^{3}$ conventional underground neutrino detector is expected to give more events. The detection of neutrinos from interactions of cosmic rays with the cosmic ray background is unfortunately only marginal for the most optimistic predictions. It should be mentioned that conventional muon underground detectors in planning will have similar difficulties, if not higher, in the detection of these neutrinos.

\section{Conclusions}

The Pierre Auger project can be made sensitive to ultra high energy neutrino fluxes through horizontal showers if an appropriate trigger is implemented. Its acceptance for detecting contained neutrino events of energy above $E_{\nu} \sim 10^{19} \mathrm{eV}$ can be very large and comparable to neutrino telescopes in planning. The highest efficiency for horizontal shower detection with the Pierre Auger Project is expected at energies about $10^{19} \mathrm{eV}$, what makes it in principle a tool for the search of the neutrinos from interactions of cosmic rays with the cosmic microwave background. The Auger array can detect the very high energy neutrinos from the decays of topological defects in some predictions. In any case, the plethora of topological defect models will be further constrained by the neutrino detection capabilities of the Auger observatories. Most recent predictions for neutrinos produced by protons accelerated in the jets of AGN have also prospects to be detected as horizontal shower events in the Auger particle arrays.

In any case, the target mass of the Auger detector is sufficiently large to serve as a significant explorating tool for ultra high energy neutrinos, whatever their source.

\section{Note added in proof}

The neutrino flux prediction TD-96 used above corresponds to a calculation by Sigl et al. as shown in Fig. 1 of Ref. [12]. For a discussion of their calculation see also G. Sigl et al., Phys. Lett. B 392 (1997) 129-134. 


\section{Acknowledgements}

We thank J. Alvarez Muñiz, J.J. Blanco Pillado, P. Billoir, F. Halzen, A. Letessier-Selvon, K. Mannheim, J. Mathews, D. Nitz, R. Protheroe, C. Pryke, G. Sigl, R. Vázquez and A.A. Watson for a number of discussions and suggestions. This work was supported in part by CICYT (AEN96-1673) and by Xunta de Galicia (XUGA-20604A96). K.S.C. was supported by NSF grant PHY 9506751 "Research Experiences for Undergraduates".

\section{References}

[1] T.K. Gaisser, F. Halzen, T. Stanev, Phys. Rep. 238 (1995) 173.

[2] V.S. Berezinsky, A.Yu. Smirnov, Astrophys. Space Science 32 (1975) 461.

[3] Design Report of the Pierre Auger Collaboration, Fermilab Report, February 1997.

[4] D.J. Thompson et al., Ap. J. Suppl. 101 (1995) 259; Ap. J. Suppl. (1996), in press.

[5] P. Biermann, P.L. Stritmatter, Ap. J. 322 (1987) 643.

[6] F.W. Stecker, M.H. Salamon, preprint astro-ph/9501064, Space Sci. Rev., to appear.

[7] A.P. Szabo, R.J. Protheroe, in: Proc. High Energy Neutrino Astrophysics Workshop, Univ. Hawaii, March 1992, V.J. Stenger, J.L. Learned, S. Pakvasa, X. Tata, eds. (World Scientific, Singapore, 1993) p. 24.
[8] J.R. Mattox et al., Ap. J. 481 (1997), to appear.

[9] K. Mannheim, Astrop. Phys. 3 (1995) 295.

[10] R.J. Protheroe, in: Proc. IAU Colloq. 163, Accretion Phenomena and Related Outflows, D. Wickramasinghe et al., eds. (1996), in press.

[11] J.J. Blanco-Pillado, R.A. Vázquez, E. Zas, Phys. Rev. Lett. 78 (1997) 3614.

[12] R.J. Protheroe, T. Stanev, Phys. Rev. Lett. 77 (1996) 3708; Erratum 78 (1997) 3420.

[13] P. Battacharjee, C.T. Hill, D.N. Schramm, Phys. Rev. Lett. 69 (1992) 567.

[14] G. Sigl et al., astro-ph/9605158, Ap. J. Lett., submitted.

[15] F.W. Stecker, C. Done, M.H. Salamon, P. Sommers, Phys. Rev. Lett. 66 (1991) 2697.

[16] S. Yoshida, M. Teshima, Prog. Theo. Phys. 89 (1993) 833.

[17] G. Parente, E. Zas, astro-ph/9606091, Proc. of the High Energy Neutrino Workshop, Venice, February 1996, p. 435.

[18] F. Halzen, Proc. of the High Energy Neutrino Workshop, Venice, February 1996, p. 499.

[19] K. Greisen, Prog. in Cosmic Ray Phys., Vol. III, J.G. Wilson, ed. (North-Holland, Amsterdam, 1956) p. 1;

K. Kamata, J. Nishimura, Prog. Theor. Phys. (Kyoto) Suppl. 6 (1958) 93.

[20] T.K. Gaisser, Cosmic Rays and Particle Physics (Cambridge Univ. Press, Cambridge, 1990), and references therein.

[21] K.S. Capelle, J.W. Cronin (1996), unpublished.

[22] A.D. Martin, R.G. Roberts, W.J. Stirling, Phys. Lett. B 387 (1996) 419;

M. Glück, E. Reya, A. Vogt, Z Phys. C Particles and Fields 67 (1995) 433.

[23] G. Parente, E. Zas, Proc. 25th Intern. Cosmic Ray Conf., Vol. 7, M.S. Potgieter et al., eds., Durban (1997) p. 109. 